\title{
The Invention of Glass, Microscopes and Telescopes : the advancement of Microbiology and Astronomy
}

\author{
by Rochelle Forrester \\ Copyright (C) 2020 Rochelle Forrester \\ All Rights Reserved \\ The moral right of the author has been asserted
}

Anyone may reproduce all or any part of this paper without the permission of the author so long as a full acknowledgement of the source of the reproduced material is made.

Second Edition

Published 2 February 2020

Preface

This paper was written in order to examine the order of discovery of significant developments in the history of glass making, microscopes and telescopes. It is part of my efforts to put the study of social and cultural history and social change on a scientific basis capable of rational analysis and understanding. This has resulted in a hard copy book How Change Happens: A Theory of Philosophy of History, Social Change and Cultural Evolution and a website How Change Happens Rochelle Forrester's Social Change, Cultural Evolution and Philosophy of History website. There are also philosophy of history papers such as The Course of History, The Scientific Study of History, Guttman Scale Analysis and its use to explain Cultural Evolution and Social Change and Philosophy of History and papers on Academia.edu, Figshare, Humanities Commons, Mendeley, Open Science Framework, Orcid, Phil Papers, SocArXiv, Social

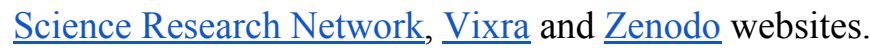

This paper is part of a series on the History of Science and Technology. Other papers in the series are:

The Invention of Stone Tools $\quad$ Fire The Neolithic Revolution $\quad$ The Invention of Pottery

History of Metallurgy The Domestication of Plants and Animals $\quad$ History of Writing

The Invention of Glass $\quad$ History of Astronomy Invention of Microscopes and Telescopes

History of Printing The Invention of the Steam Engine History of Electricity

Electric Telegraph $\underline{\text { Telephone }} \underline{\text { Radio }} \underline{\text { Television }} \underline{\text { Photography }} \underline{\text { Motion Pictures }}$

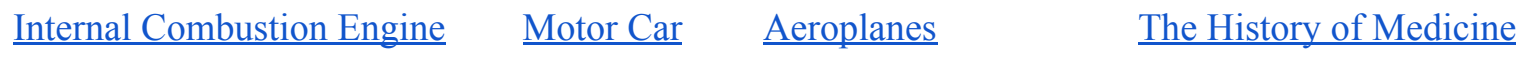

The Discovery of the Periodic Table The Discovery of the Subatomic Particles

Other papers by Rochelle Forrester include works on Epistemology and the Philosophy of Perception such as Sense Perception and Reality and on quantum mechanics such as the Quantum Measurement Problem and The Bohr and Einstein debate on the meaning of quantum physics. Rochelle Forrester's work is also published on Slideshare, Issuu and Scribd. Rochelle Forrester is a member of the International Network for Theory of History. 


\begin{abstract}
The ultimate cause of much historical, social and cultural change is the gradual accumulation of knowledge of the human environment. The invention of glass and microscopes and telescopes, substantially increased human knowledge of the human environment. Microscopes and telescopes would not have been possible without the prior discovery of glassmaking and some knowledge of the law of refraction. Without the microscope we would have no knowledge of micro organisms and the causes of many diseases. Without the telescope we would probably still believe the sun and planets orbited a stationary earth. The change from an earth centered universe, the common sense theory for societies with unassisted vision, to a sun centered theory such as the Newtonian system, and then to General Relativity was inevitable. This was because the knowledge provided by unassisted vision, naturally led to an earth centered universe, the knowledge available from 17 th to 19 th century telescopes and mathematics led naturally to a sun centered system, such as the Newtonian system and better telescopes led naturally to a theory such as General Relativity. The human environment has a particular structure so that human knowledge of the environment is acquired in a particular order. The simplest knowledge is acquired first and more complex knowledge is acquired later. The order of discovery determines the course of human social and cultural history as knowledge of new and more efficient means of meeting human needs, results in new technology, which results in the development of new social and ideological systems. This means human social and cultural history, has to follow a particular course, a course that is determined by the structure of the human environment.
\end{abstract}

Glass

The earliest known glass making was in ancient Egypt around 3,000 BCE, although Mesopotamia followed quickly thereafter. The basic requirements for making glass are silica (sand), an alkaline substance such as soda, and lime (calcium carbonate). The earliest and simplest technique for glass manufacture was baked glazing. Baked glazing involved melting the silica, soda and lime and then coloring the product which was then poured into molds to produce small statutes or jewelry. It was later discovered that a bottle or vase could be made by placing the molten glass around a core that could be removed when the glass cooled.

In the first century CE glass blowing was developed probably in the area of present day Syria. Glass blowing involved the molten glass being placed at the end of a metal pipe and a person blowing through the pipe which blew the glass up to the desired size and then the glass was shaped and decorated. The development of glass blowing opened up new technical and artistic possibilities for glass makers. The quality of the glass improved as its texture became more refined and it became more transparent and colorless. New molding techniques developed with the glass being blown into the molds. Glass began to be used to make plates, pitchers and 
vases, objects which had previously been made mainly of metal or clay. The Roman author Pliny referred to the use of manganese oxide to rid glass of impurities so as to ensure that it was transparent.

Chemically glass is an amorphous non crystalline solid. This means the atoms are arranged in a random rather than a regular pattern. This accounts for the optical qualities of glass such as transparency and causes the glass to not have a definite melting point. When heated the silicon in glass causes it to go into a state where it is a soft solid, or viscous liquid. This state exists over a wide temperature range and explains why glass is able to be molded and blown into a wide variety of shapes.

If it had not been possible to invent glass, for example if no combination of materials could produce a solid transparent substance, then the effect on society would have been considerable. Eye glasses to correct defective vision would have been impossible and the microscope and the telescope may not have been invented. This would have meant our discovery of the world of microorganisms may not have happened or may have been delayed until the 20th century and the development of other transparent products such as plastics. Progress in medical research and the discovery of disease would have been delayed or never happened. It would also have meant that our discovery of the universe from new planets to new galaxies would have been delayed until other transparent materials could have been developed. The Earth centered astronomies of the pre-telescope age would have continued to be believed at least until some substitute for glass had been developed and could be used to create telescopes.

\section{Microscopes}

The transparency of glass combined with the way in which light changes direction as it passes through one transparent medium to another, a phenomenon known as refraction, allows glass to be used for the purposes of magnification. When two (or with poorer results, one) lenses are used an object seen through those lenses is magnified. This is because the shape of the lenses causes light going through the lenses to converge at a particular focus or focal point in accordance with the laws of refraction. This focal point is different from the normal human focal point and allows the object to be magnified without blurring. The apparent size of an object increases as it is brought closer to the eye, but if it is brought to close, blurring occurs. The blurring occurs because the lens in our eye cannot bend (or refract) light from an object enough to bring it into proper focus on the retina, if it is to close. The lenses magnify by starting the refraction, or bending process, before the light enters the eye. This enables objects closer than the usual human focal point to be examined without losing focus. This was to result in the invention of glasses to correct bad vision and in the invention of the telescope and the microscope. 
The particular shape of the lenses used in microscopes can be worked out by using the law of refraction (known as Snel's law after Willebrod Snel (1580-1626)) and trigonometry which was developed by the mathematician Rheticus (1514-76). An index of refraction establishes the angle at which light bends when going from one medium to another. When light passes from air through glass, the refractive index is approximately 1.52. Armed with this knowledge it is possible to manufacture both microscopes and telescopes. Alternatively the earliest microscopes and telescopes may have been developed simply by experiment and observation.

The earliest lenses produced were eye glasses to correct defective vision and these were first developed in 13th century Italy. They were clearly developed from experimentation and observation without the benefit of Snel's law or trigonometry. The earliest microscopes were invented by the Dutch spectacle makers Hans and Zacharus Janssen about 1590.

The social and cultural consequences of the invention of the microscope, was the discovery of whole new worlds. An immense variety of microorganisms were discovered, the leading microscopist being Antoni van Leeuwenhoek (1632-1723). Van Leeuwenhoek discovered protozoa in water, bacteria, blood corpuscles, capillaries, striations in skeletal muscle, the structure of nerves and spermatozoa. The microscope was soon to destroy the idea of spontaneous generation which held that many animals arose from spontaneous generation from particular environments. Mosquitoes came from stagnant water, bees from the carcasses of oxen and cattle, shellfish from mud and slime and snails from the putreification of fallen leaves. The work of van Leeuwenhoek and others showing the life style and sexual apparatus of such animals showed the idea of spontaneous generation was wrong, although it was not until the 19th century with the work of Louis Pasteur that the idea was finally put to an end.

The 17th and 18th century microscope had its thresholds so that while it could reveal certain things previously unknown, there was much it could not reveal. This lead to some theories that would not last due to more powerful microscopes proving them wrong. One such theory was that spermatozoa was the essential instrument of reproduction which fitted in with the belief in the pre-existence of organisms. Each new organism was considered to contain all the characteristics of all its predecessors. The spermatozoon was considered to be the means of transmission of all those characteristics to the new organism. This idea however failed to understand the role of the spermatozoon in fertilizing the egg and the contribution of the egg to the characteristics of the new organism. The idea however, based on the information available too 17 th and 18th century scientists, was reasonable enough for the times. It was not until the 19th century when improved microscopes showed the spermatozoon and the egg contributed equally to the characteristics of the new organism. 
Telescopes

The telescope appears to have been invented by Hans Lipperhey, a spectacle maker in the Dutch town of Middelburg, who applied for a patent for it, in 1608. Two other Dutchmen, Jacob Adriaenzoon and Sacharias Janssen also claimed to have invented the telescope, so a patent was refused. The Italian scientist Galileo heard about the Dutch invention and constructed his own telescope achieving a magnification of 20x, a better magnification than was to be achieved until 1630. Galileo's telescope had two lenses, an objective lense at one end of the telescope and an ocular lense at the other end, to which the eye was applied. The objective lense was a convergent or biconvex lense while the ocular lense was a divergent or biconcave lense. The effect of light passing through the lenses was to change the focal point of the light providing for a wider visual angle in which to view the object under observation. The telescope, while operating a bit differently from a microscope, like the microscope, magnifies images through manipulating the focal point of light to create a wider visual angle in accordance with the laws of refraction.

Galileo, having created his telescope used it to look at the sky. He discovered a large number of previously unseen stars (the Milky Way), that the moon had an irregular surface, the sun was spotty and impure (sunspots), that Jupiter had four moons, there were rings around Saturn and the moon like phases of Venus. The observations were contrary to the astronomical theories of Ptolemy, which had largely been accepted from classical times. Galileo's observations plus those of other scientists using even better telescopes were to result in the ending of the Ptolemaic astronomy and its eventual replacement with the Newtonian system.

Prior to the invention of the telescope six planets (the Earth itself, Mercury, Venus, Mars, Jupiter and Saturn) were known to human beings and less than 5,000 stars were visible to the naked eye. The telescope led to the discovery of Uranus in the 18th century, Neptune in the 19th century and Pluto in the 20th century. The invention of photography assisted the telescope in revealing the universe as it allowed objects to dim to be seen through a telescope to be photographed on a photographic plate over a long exposure time. The long exposure time allowed the photographic plate to record the existence of very faint objects as the plate will accumulate the effect of each photon hitting the plate over a period of time.

By the start of the 20th century it had become clear that our solar system was part of the Milky Way, but it was not clear whether the Milky Way was the whole universe. It was not until the 1920's when Edwin Hubble conclusively showed there were other galaxies and these galaxies were moving away from us, with the furthest galaxies moving the fastest.

New forms of telescopes, which detected different forms of electromagnetic energy, were developed. However, most electromagnetic energy, other than visible light and radio waves, is blocked by the earth's atmosphere. The development of space rockets lead to telescopes being placed in space particularly the Hubble space telescope to allow detection of electromagnetic 
radiation in frequencies other than those of visible light and radio waves. Telescopes using frequencies other than those of visible light have detected radio wave evidence of planets in other solar systems, $\mathrm{x}$-ray evidence of black holes, radio-wave evidence of supernova explosions, and gamma ray and $\mathrm{x}$-ray evidence of gamma rays originating from deepest space. Dark matter that could not be detected by any telescope operating on any electromagnetic wavelength was detected due to its gravitational effect on matter that was visible to telescopes.

\section{Bibliography:}

Cardwell, Donald (1994) The Fontana History of Technology, Fontana Press, London Crump, Thomas (2001) A Brief History of Science, Robinson, London

Daumas, Maurice (ed) (1964) A History of Technology and Invention, Presses Universitaires de France

Fergusson, Kitty (1999) Measuring the Universe, Headline Book Publishing, London

Gribben, John (2002) Science: A History, 1543-2001, Penguin Books, London

Meyer, Jerome (1956) World Book of Great Inventions, The World Publishing Company, New York

Sagan, Carl (1984) Cosmos, Futura, London \& Sydney

Silver, Brian L (1998) The Ascent of Science, Oxford University Press, Oxford

Singh, Simon (2005) Big Bang, Harper Perennial, London

Taylor, Gordon Rattray (1983) The Inventions that Changed the World, Reader's Digest, London 